\title{
On the origin of nonlocal damping in plasmonic monomers and dimers
}

Tserkezis, Christos; Yan, Wei; Hsieh, Wenting; Sun, Greg; Khurgin, Jacob B.; Wubs, Martijn; Mortensen, N. Asger

Published in:

International Journal of Modern Physics B

Link to article, DOI:

$10.1142 / S 0217979217400057$

Publication date:

2017

Document Version

Peer reviewed version

Link back to DTU Orbit

Citation (APA):

Tserkezis, C., Yan, W., Hsieh, W., Sun, G., Khurgin, J. B., Wubs, M., \& Mortensen, N. A. (2017). On the origin of nonlocal damping in plasmonic monomers and dimers. International Journal of Modern Physics B, 31(24), [174005]. https://doi.org/10.1142/S0217979217400057

\section{General rights}

Copyright and moral rights for the publications made accessible in the public portal are retained by the authors and/or other copyright owners and it is a condition of accessing publications that users recognise and abide by the legal requirements associated with these rights.

- Users may download and print one copy of any publication from the public portal for the purpose of private study or research.

- You may not further distribute the material or use it for any profit-making activity or commercial gain

- You may freely distribute the URL identifying the publication in the public portal 


\title{
ON THE ORIGIN OF NONLOCAL DAMPING IN PLASMONIC MONOMERS AND DIMERS
}

\author{
CHRISTOS TSERKEZIS \\ Department of Photonics Engineering, Technical University of Denmark, \\ DK-2800 Kongens Lyngby, Denmark \\ ctse@fotonik.dtu.dk \\ WEI YAN
}

Laboratoire Photonique, Numérique et Nanosciences (LP2N), IOGS Univ. Bordeaux CNRS, 33400 Talence cedex, France

WENTING HSIEH

Department of Physics, University of Massachusetts, Boston, Massachusetts 02125, USA

\section{GREG SUN}

Department of Engineering, University of Massachusetts, Boston, Massachusetts 02125, USA

JACOB B. KHURGIN

Department of Electrical and Computer Engineering, Johns Hopkins University, Baltimore, Maryland 21218, USA

\section{MARTIJN WUBS}

Department of Photonics Engineering, Technical University of Denmark, DK-2800 Kongens Lyngby, Denmark

Center for Nanostructured Graphene, Technical University of Denmark, DK-2800 Kongens Lyngby, Denmark mwubs@fotonik.dtu.dk

\section{N. ASGER MORTENSEN}

Center for Nano Optics, University of Southern Denmark, Campusvej 55, DK-5230 Odense M, Denmark

Center for Nanostructured Graphene, Technical University of Denmark, DK-2800 Kongens Lyngby, Denmark

Department of Photonics Engineering, Technical University of Denmark, DK-2800 Kongens Lyngby, Denmark

asger@aps.org

Received Day Month Year

Revised Day Month Year 
The origin and importance of nonlocal damping is discussed through simulations with the generalized nonlocal optical response (GNOR) theory, in conjunction with timedependent density-functional-theory (TDDFT) calculations and equivalent circuit modeling, for some of the most typical plasmonic architectures: metal-dielectric interfaces, metal-dielectric-metal gaps, spherical nanoparticles, and nanoparticle dimers. It is shown that diffusive damping, as introduced by the convective-diffusive GNOR theory, describes well the enhanced losses and plasmon broadening predicted by $a b$ initio calculations in few-nm particles or few-to-sub-nm gaps. Through the evaluation of a local effective dielectric function, it is shown that absorptive losses appear dominantly close to the metal surface, in agreement with TDDFT and the mechanism of Landau damping due to generation of electron-hole pairs near the interface. Diffusive nonlocal theories provide therefore an efficient means to tackle plasmon damping when electron tunneling can be safely disregarded, without the need to resort to more accurate, but time-consuming fully quantum-mechanical studies.

Keywords: Nonlocal optical response; plasmon damping; convective-diffusive theory.

\section{Introduction}

Following advances in nanofabrication, the research field of plasmonics has developed on the foundation of classical electrodynamics and semiclassical descriptions of light-matter interactions. ${ }^{1,2,3}$ In most cases, the collective oscillations of free electrons subject to optical fields are conceptually analyzed within the Drude theory, ${ }^{4}$ inherently using the local-response approximation (LRA). Within this picture, the material response occurs only at the spatial position of the perturbation, while there is not even the slightest response at any short range away. ${ }^{5}$ For dielectric media, this is a well-established and accurate approach, while metals in principle support short-range correlations that are potentially important to true nanoscale plasmonics and applications in the field of quantum plasmonics. ${ }^{6,7,8,9}$ Despite its simplifications, the LRA framework has fostered both theory predictions and experimental confirmations of a large range of plasmonic phenomena, such as confining of light beyond the diffraction limit ${ }^{10,11}$ and tuning the optical properties of metallic architectures through size and shape variations. ${ }^{12}$ Large enhancement of electric fields in the vicinity of metal nanostructures ${ }^{13,14,15}$ is another interesting plasmonic property, where abrupt variations in metal-surface topology may support huge amplifications. Such field enhancement is intimately linked to field singularities, which are inherent to LRA.

Accounts of light-matter interactions commonly rely on linear-response theory. For dielectric materials the further simplification associated with LRA usually holds all the way down to the atomic scale. The success of LRA is in some way more intriguing in the case of nanoplasmonics. On the one hand, experimental characterization of gold dimers with narrow, few-nm gaps has been found to agree well with classical electrodynamics. ${ }^{16}$ On the other hand, when light interacts with the free electrons in metals, the optical response is anticipated to exhibit a nonlocal character, with the corresponding response function becoming spatially dispersive. ${ }^{5}$

The underlying quantum wave dynamics of the electron gas manifests itself at a length scale intrinsic to the metal: the Fermi-wavelength scale. For most metals, this 
lies in the nanometer-to-Angstrom scale regime. ${ }^{4}$ Such a small scale can therefore justify the success of LRA and the application of Drude theory to plasmonics even in nanoscale metallic structures. At the same time, it also hints to the mesoscopic size regime for which a departure from the predictions of classical electrodynamics can be anticipated. This expectation has been confirmed, for example, for arbitrarily sharp changes in the metal-surface topography and in dimers with vanishing gaps, where disregarding nonlocality causes the field to diverge within the LRA response. ${ }^{15,17}$

Here we review aspects of the nonlocal response of metallic nanoparticles, which was recently revived ${ }^{18,19,20,21}$ by the realization of state-of-the-art experimens on plasmonics in structures of ever smaller dimensions. ${ }^{22,23,24,25,26,27,28,29,30,31}$ In particular, we first explore the real-space formulation ${ }^{21,32}$ and numerical implementations ${ }^{33}$ of the long-existing nonlocal hydrodynamic theory. ${ }^{34,35,36,37} \mathrm{We}$ then address a recent extension of this theory which accounts for drift-diffusion dynamics, namely the generalized nonlocal optical response (GNOR) theory. ${ }^{38}$ By extracting an effective local dielectric function of the metal from GNOR simulations, and in comparison with time-dependent density functional theory (TDDFT) calculations for metal-dielectric interfaces, we show that the increased plasmon damping in small nanoparticles and narrow gaps can be fully understood in terms of inducedcharge diffusion, thus providing a connection between Landau damping and the nonlocal optical response. ${ }^{39,40,41,42}$

\section{Nonlocal electrodynamics}

Theoretical modeling of plasmonic phenomena widely relies on the macroscopic Maxwell's equations. ${ }^{1}$ Here, the optical response of metals is described through constitutive relations connecting the response of the material to the applied field. In particular, the displacement field $\mathbf{D}$ generated at a point $\mathbf{r}$ in response to a perturbing electric field $\mathbf{E}$ is in general given by the nonlocal constitutive relation

$$
\mathbf{D}(\omega, \mathbf{r})=\varepsilon_{0} \int d \mathbf{r}^{\prime} \varepsilon\left(\omega, \mathbf{r}, \mathbf{r}^{\prime}\right) \mathbf{E}\left(\omega, \mathbf{r}^{\prime}\right)
$$

where $\varepsilon_{0}$ is the vacuum permittivity, $\omega$ is the angular frequency of light and $\varepsilon$ is the corresponding (nonlocal) permittivity of the metal. Introducing Eq. (1) into Maxwell's equations one arrives at the integro-differential wave equation

$$
\nabla \times \nabla \times \mathbf{E}(\omega, \mathbf{r})=\left(\frac{\omega}{c}\right)^{2} \int d \mathbf{r}^{\prime} \varepsilon\left(\omega, \mathbf{r}, \mathbf{r}^{\prime}\right) \mathbf{E}\left(\omega, \mathbf{r}^{\prime}\right),
$$

where $c$ is the velocity of light in vacuum. The above equation is not particularly appealing, as it is not straightforward to solve it, either analytically or numerically. However, if one focuses on short-range correlations, it can be transformed into a regular partial differential equation ${ }^{32,38,43}$

$$
\nabla \times \nabla \times \mathbf{E}(\omega, \mathbf{r})=\left(\frac{\omega}{c}\right)^{2}\left[\varepsilon_{\mathrm{LRA}}(\omega)+\xi^{2} \nabla(\nabla \cdot)\right] \mathbf{E}(\omega, \mathbf{r}) .
$$


Here, $\xi$ gives the range for the nonlocal response, while $\varepsilon_{\mathrm{LRA}}$ is the usual Drude contribution. Within GNOR hydrodynamics, the nonlocal length scale is given by ${ }^{38}$

$$
\xi^{2}=\frac{\beta^{2}}{\omega(\omega+\mathrm{i} \gamma)}+\frac{D}{\mathrm{i} \omega}
$$

where $\beta^{2}=3 / 5 v_{\mathrm{F}}^{2}$ with $v_{\mathrm{F}}$ being the Fermi velocity, while $D \propto v_{\mathrm{F}}^{2} \tau$ is a diffusion constant associated with impurity scattering, $\tau$ is the free-electron relaxation time, and $\gamma=1 / \tau$ is the usual Drude damping rate. ${ }^{32,38}$ Setting $D=0$ in Eq. (4), one immediately retrieves the standard nonlocal hydrodynamic Drude model (HDM), which predicts modal blueshifts but no additional broadening. ${ }^{32}$

To get more physical insight into the role of the diffusion term in the hydrodynamic description of the electron gas, the relaxation dynamics has to be considered. Under the influence of an external electric field, the free electrons in an initially charge-neutral metallic nanostructure are drawn away from their equilibrium positions, leaving regions near the surfaces with excess and deficit densities of charge, i.e. an induced net surface charge. At the same time, of course, the bulk remains uncharged, since the positive ions remain fully screened by a corresponding density of negatively charged conduction electrons. As a consequence of entropy, the induced spatially inhomogeneous charge density will relax towards its equilibrium distribution, a relaxation driven by surface scattering and many-body electron-electron interactions. Classically, this is described as diffusion and in a drift-diffusion theory this is captured by the diffusion constant $D$. Since diffusion relaxes the induced charge, it also relaxes the polarization field, and consequently it is a damping mechanism.

\section{Bridging the nonclassical response of monomers and dimers}

Individual metallic nanoparticles, and their dimer counterparts, consist the archetypal plasmonic structures. In the following we show that GNOR is versatile enough to offer an understanding of enhanced damping mechanisms in both. Several attempts to tackle this issue have been presented over the years. Among the pioneers, Kreibig and co-workers presented a phenomenological model (referred to as SDB in what follows) which successfully captures the size-dependent spectral broadening observed in few-nm, nearly-spherical nanoparticles. ${ }^{44}$ Apart from its phenomenological character, the main disadvantage of this approach is the absence of simple guidelines on how to apply it to non-spherical monomers or to strongly interacting dimers composed of particles that are not necessarily small themselves. In a recent effort to deal with nonclassical effects in dimers, Esteban et al. developed a quantum-corrected model ${ }^{45}$ which introduces quantum tunneling as the main driving force behind the dimer nonclassical optical response. Tunneling-based theories, however, can naturally not address the nonclassical aspects of the optical response of the corresponding monomers. This is where GNOR proves versatile and with a predictive power reaching far beyond either limiting case described above. Despite its simplicity and semi-classical nature, GNOR provides a unified description 


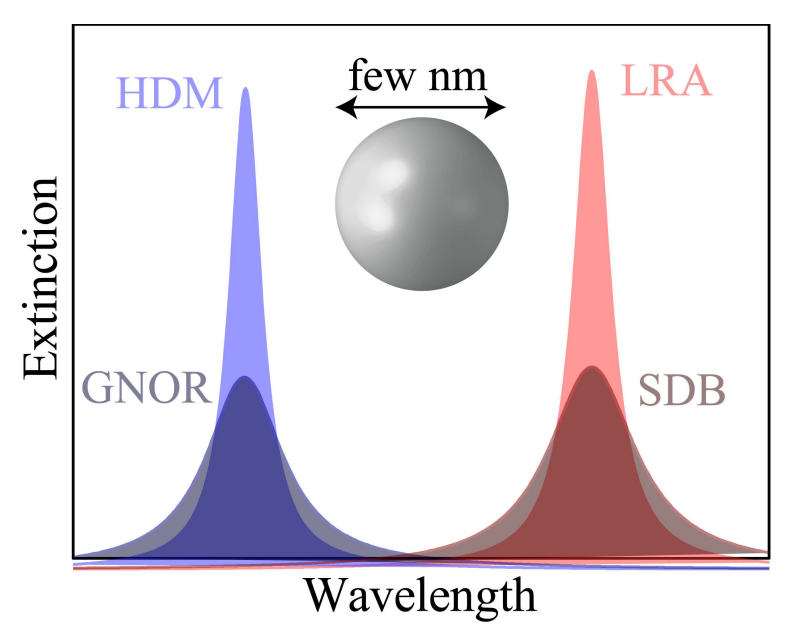

Fig. 1. Schematic representation of typical extinction spectra calculated within the LRA (light red), SDB (dark red), HDM (light blue), and GNOR (dark blue) models, for a few-nm plasmonic nanosphere.

of both size-dependent damping in monomers and gap-dependent broadening in dimers, and stresses the connection between the two, simply through the addition of a diffusive term to standard hydrodynamics.

Let us first briefly discuss the case of a monomer. We consider a metallic nanosphere of radius $R$, for which the complex-valued nonlocal length scale $\xi$ in Eq. (4) leads to $1 / R$ corrections to both the dipole resonance frequency and to its linewidth. ${ }^{38}$ This observation provides an immediate link between the diffusion constant $D$ and the $A$ coefficient appearing in the approach developed by Kreibig et $a l .,{ }^{44}$ in which the Drude damping rate $\gamma$ is corrected by adding the term $A v_{\mathrm{F}} / R$. For most metals, $A$ is experimentally found to be of order unity. This plasmon broadening, and the way it is captured by the SDB and GNOR models, is schematically depicted in Fig. 1, where we show typical extinction spectra for a few-nm metallic nanoparticle within the LRA, SDB, HDM and GNOR models. A more detailed description of the differences between the four models and their application to different types of metals and statistical ensembles of small, weakly interacting nanoparticles can be found in Ref. 46 .

In a similar manner, it has also been shown that the very same choice of the diffusion constant $D$ can qualitatively explain the gap-dependent broadening of spectra for dimers as well. ${ }^{38}$ So, what is the important common feature of monomers and dimers? Can the experimentally observed broadening ${ }^{24,47}$ be attributed exclusively to a short-circuiting of the capacitive junction due to tunneling - as described in the quantum-corrected model ${ }^{45}$ and its more recent extensions? ${ }^{48,49}$ Or could diffusive damping - for which we will give a microscopic explanation below - account for it? Of course, there is no reason why these mechanisms could not co-exist and act 
simultaneously.

To facilitate a qualitative discussion we employ a circuit model that addresses the relative importance of diffusive damping (characterized by a resistance $R_{\text {dif }}$ ) and the damping associated with the relaxation of the quantum tunneling current (characterized by $R_{\text {tun }}$ ) short-circuiting the classically impenetrable capacitive gap (characterized by a capacitance $C$ ). Within this equivalent circuit model, $C$ and $R_{\text {tun }}$ are connected in parallel to describe the plasmonic gap, ${ }^{50,51}$ and this branch is then connected in series with $R_{\text {dif }}$. The equivalent impedance is given by

$$
Z=R_{\text {dif }}+\frac{R_{\text {tun }}}{1+\mathrm{i} \omega \tau_{\text {tun }}}=R_{\text {dif }}-\frac{\mathrm{i}}{\omega C}+\mathcal{O}\left[1 /\left(\omega \tau_{\text {tun }}\right)^{2}\right],
$$

where $\tau_{\text {tun }}=R_{\text {tun }} C$ is interpreted as the tunneling RC time..$^{50}$ This RC model has been successful in analyzing the ultra-fast response of a scanning-tunneling microscope ${ }^{50}$ Here, we extended it to include dissipation associated with the diffusive dynamics.

The tunneling dynamics simplifies in the slow adiabatic-following regime and the limit of fast external driving. ${ }^{52}$ The high-frequency dimer dynamics is therefore entirely dominated by the diffusive broadening and the junction capacitance, see the second equality in Eq. (5). In the context of the mesoscopic capacitance, ${ }^{53}$ ultra-fast tunneling experiments have reported tunneling RC times that lie in the picosecond range, ${ }^{50,54}$ implying that at optical frequencies the plasmon response may just be too fast. If this is the case, $\omega \tau_{\text {tun }} \gg 1$, and the relaxation will be dominated by diffusive broadening.

The above circuit analysis does not depend on the exact tunneling mechanism. Whether tunneling relaxation occurs within the gap, as assumed in the quantumcorrected model ${ }^{45}$ or inside the metal surfaces, as in the model by Hohenester ${ }^{49}$ (in agreement with the common understanding of relaxation processes in mesoscopic quantum electron transport), is not relevant here, and the conclusions are unaltered. Nevertheless, it should be stressed that, given the qualitative nature of the above analysis, and the lack of conclusive data for the RC time in plasmonic dimers, the situation remains still open. In fact, for few-Angstrom gaps a different, shorter RC time might be reasonable, in which case tunneling shall indeed become important. ${ }^{42,55}$

\section{Connection between diffusion and Landau damping}

The SDB correction introduced by Kreibig et al. has already been linked to quantum mechanical calculations of Landau damping associated with surface-enhanced electron-hole pair generation in the metal. ${ }^{39,56,57}$ More recently, ab initio studies ${ }^{42}$ and electron spectroscopy ${ }^{30,58,59}$ have established plasmon damping exceeding the expectations based on bulk material parameters at the very surface of metals. The underlying many-body interaction within the electron gas is captured by GNOR with one single and entirely classical parameter: the diffusion constant $D$. Since the induced charge resides near the metal surface, this is where diffusion is also effective, 

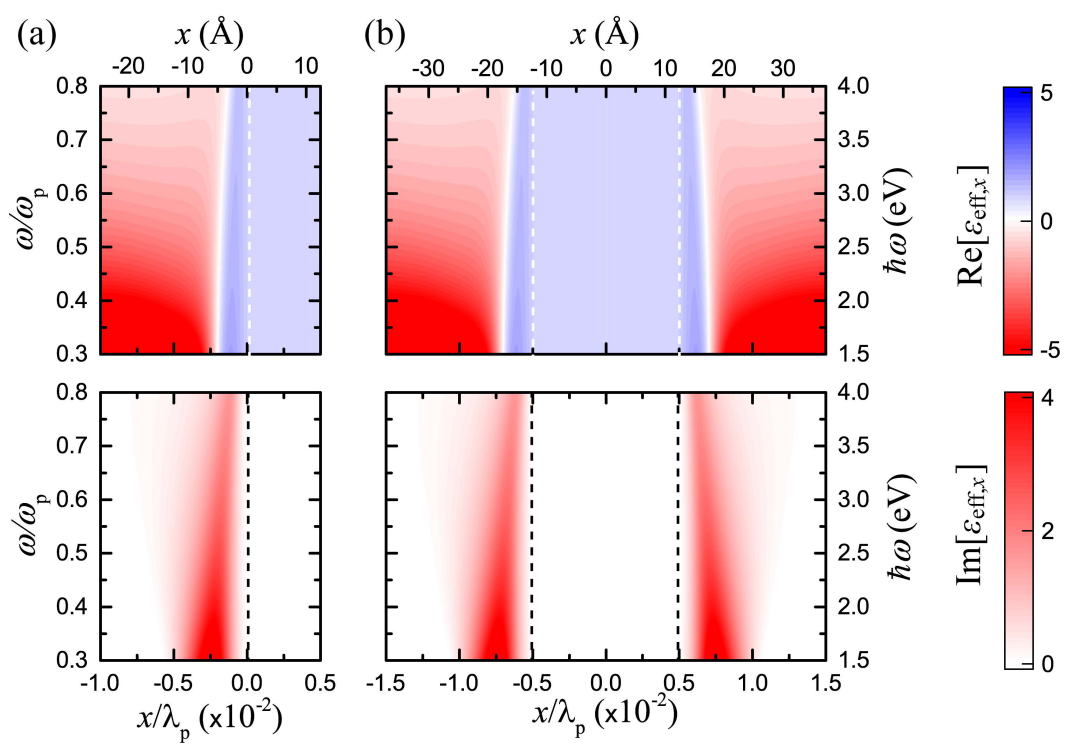

Fig. 2. GNOR results for the real part (top panels) and imaginary part (lower panels) of $\varepsilon_{\text {eff }, x}$ (where $x$ corresponds to the direction normal to the interface) for different frequencies (normalized to the metal plasma frequency $\omega_{\mathrm{p}}$ ) throughout the optical range, as a function of distance $x$ (normalized to the plasma wavelength $\lambda_{\mathrm{p}}=2 \pi c / \omega_{\mathrm{p}}$ ). (a) A single metal-air interface with an infinite work function and a homogeneous equilibrium electron density that vanishes abruptly outside the metal surface. (b) Corresponding metal-air-metal cavity with a $0.01 \lambda_{\mathrm{p}}$ gap separating the two surfaces. Right and top axis scales give the corresponding values in energy and length, assuming a plasma energy $\hbar \omega_{\mathrm{p}}=5 \mathrm{eV}$. Dashed lines denote the metal-air interfaces.

as it is shown by the nonlocal correction term in Eq. (3), which mainly contributes near the surface where the $\mathbf{E}$ field changes most. This is why our drift-diffusion model mimics both longitudinal pressure waves and Landau-like damping so well. The enhanced damping near the surface is illustrated in Fig. 2 where we show the local effective permittivity $\varepsilon_{\text {eff }, x}(\mathbf{r}, \omega)$ (the component normal to the metal-air interface, $x$ axis $)$ extracted from GNOR simulations $\left(\gamma=0.0023 \omega_{\mathrm{p}}, v_{\mathrm{F}}=0.0045 c\right.$, $\left.D=2 \omega_{\mathrm{p}} / c^{2}\right)$ via $\mathbf{D}(\mathbf{r}, \omega) \equiv \varepsilon_{0} \varepsilon_{\text {eff }}(\mathbf{r}, \omega) \mathbf{E}(\mathbf{r}, \omega)$, for either a flat metal-air interface (a) or a narrow metal-air-metal cavity (b). It is important to notice here that, due to the abrupt termination of the surface and the associated hard-wall boundary condition for the normal component of the current, the additional damping occurs slightly inside the surface. This links up to the Feibelman parameter ${ }^{60}$ and its associated quantum corrections to electrodynamics. ${ }^{61}$ The importance of relaxing the hard-wall boundary condition and considering the actual position of the surface of the electron plasma was recently discussed by Teperik and co-workers ${ }^{62,63}$ in the context of plasmonic ruler effects of sub-nm gap dimers. In the spirit of our current discussion, it has recently been shown that density-gradient and spill-out effects can also be included in self-consistent hydrodynamic models. ${ }^{64,65,66}$

The role of Landau damping for both monomers and dimers can be pictorially 
(a)
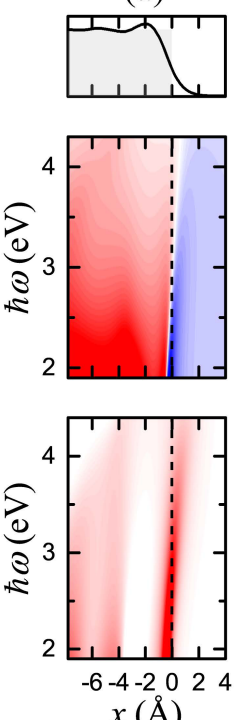

$x(\AA)$ (b)
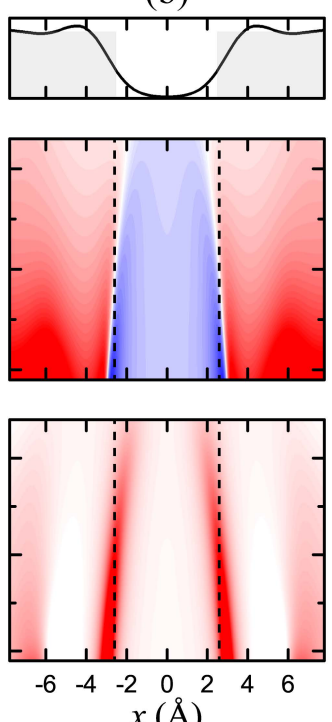

$x(\AA)$ (c)
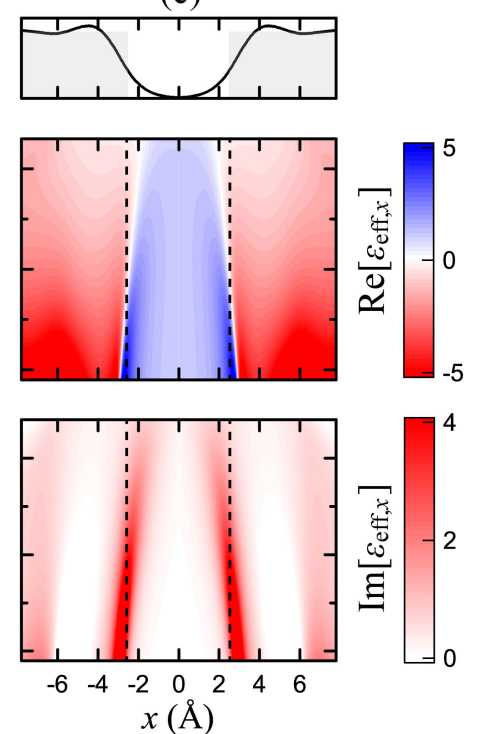

Fig. 3. Equilibrium density in the jellium model (top panels), exhibiting both Friedel oscillations and electron spill-out, along with the TDDFT results for the real part (middle panels) and imaginary part (lower panels) of $\varepsilon_{\mathrm{eff}, x}$ as a function of frequency. (a) A single Air-Na interface. (b) Full TDDFT calculation for two interacting Na surfaces separated by a 0.5-nm air gap. (c) The same metal-air-metal gap as in (b), with the results obtained by a superposition of two of the independent surfaces shown in (a). All contours share a common color scale.

illustrated by the time-dependent optical response of the electron gas near the surface of the metal. ${ }^{42}$ We consider a simple metal, such as $\mathrm{Na}$, described within a jellium approximation, and exploit TDDFT to obtain its response to a timedependent electrical field. ${ }^{42,62}$ The calculation provides both the (space dependent) equilibrium density $n_{0}(\mathbf{r})$ and the induced charge density $n_{1}(\mathbf{r})$. Additionally, one also obtains the displacement field $\mathbf{D}$ generated by the perturbing $\mathbf{E}$ field, and from this one may again infer an effective relative dielectric function $\varepsilon_{\text {eff }}(\mathbf{r}, \omega)$, where the imaginary part holds key information about damping and its spatial localization. The top panel of Fig. 3(a) illustrates the equilibrium density (exhibiting both Friedel oscillations and quantum spill-out) along with the real part (middle panel) and imaginary part (lower panel) of $\varepsilon_{\text {eff, } x}$ as a function of frequency. Observing the imaginary part of $\varepsilon_{\text {eff, } x}$, one can immediately notice how large Landau damping (exceeding the bulk damping) takes place in the near vicinity of the surface. We also note that this large imaginary part of $\varepsilon_{\mathrm{eff}, x}$ is always accompanied by a change in sign for the real part, which denotes the presence of a metal-dielectric interface, and is the prerequisite for plasmon excitation. In addition, we note that the large imaginary part regularizes the field enhancement driven by the vanishing real part, as first explored by Öztürk et al. ${ }^{67}$ 

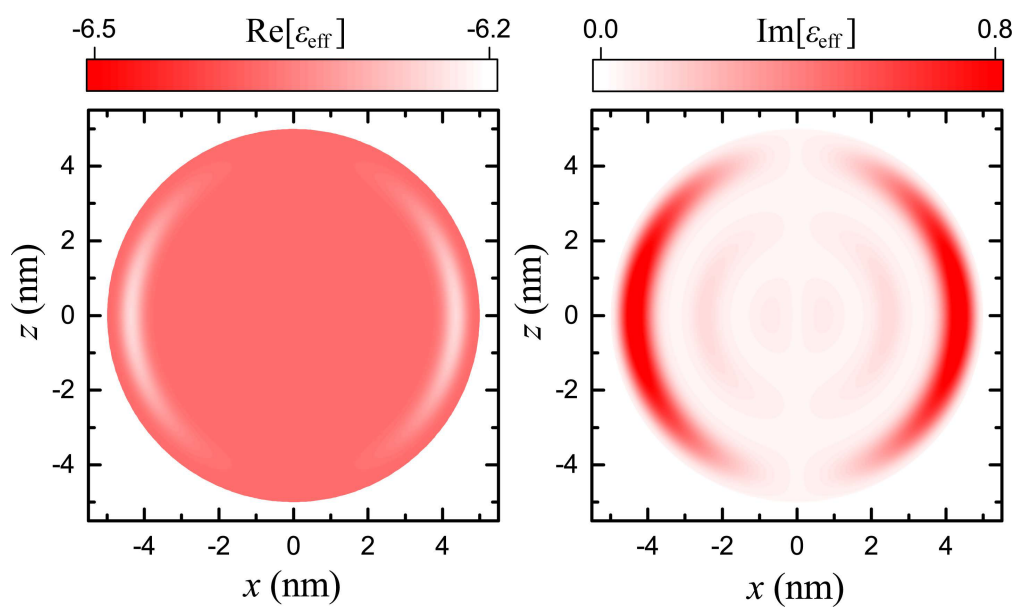

Fig. 4. Real (left-hand contour) and imaginary (right-hand contour) part of the effective dielectric function, $\varepsilon_{\text {eff }}$, calculated at the resonant wavelength $(\lambda=370 \mathrm{~nm})$ for a Drude nanosphere $\left(\varepsilon_{\mathrm{b}}=\right.$ $\left.4.1, \hbar \omega_{\mathrm{p}}=9.3 \mathrm{eV}, \hbar \gamma_{\mathrm{b}}=0.013 \mathrm{eV}, \hbar \gamma_{\mathrm{s}}=0.83 \mathrm{eV}\right)$ of radius $R=5 \mathrm{~nm}$, calculated according to Eq. (6).

The same calculation can be done for dimers as well. In the one-dimensional example studied above, this corresponds to two opposing jellium surfaces separated only by a sub-nm gap. The corresponding calculation for a 0.5-nm gap is shown in Fig. 3(b), where we recover the physical picture obtained for the single interface. Significant Landau damping occurs now at both interfaces, while there is no appreciable damping inside the gap. In fact, the response of the dimer is well represented by a simple superposition of the response of two opposing independent surfaces, see Fig. 3(c), thus confirming the suggestion of the circuit analysis: even for a gap of 0.5 $\mathrm{nm}$, the dissipation is dominated by Landau damping, and tunneling currents do not change the plasmon-energy dissipation. We note that the typical ab initio simulations of the electron-gas dynamics include no energy-relaxation processes (e.g. coupling to a thermalizing phonon bath) and as such tunneling currents are necessarily relaxed only through the generation of electron-hole pairs. We emphasize that the observed importance of Landau damping does not rule out the existence of quantum tunneling currents at optical frequencies; in the present context of $0.5-\mathrm{nm}$ gaps such currents start to appear, but they do not significantly influence the gapdependent broadening of dimers yet. One needs to enter the true Angstrom-scale regime, where the individual atoms start to matter.

To further advance our description, we shift attention from flat interfaces to single metallic nanoparticles. In addition to the methods described above, the effective dielectric function can also be calculated by adding up in the Drude expression the bulk $\left(\gamma_{\mathrm{b}}\right)$ and surface broadening damping rate $\left[\gamma_{\mathrm{s}}(r)\right]$, according to Matthiesen's 
(a)
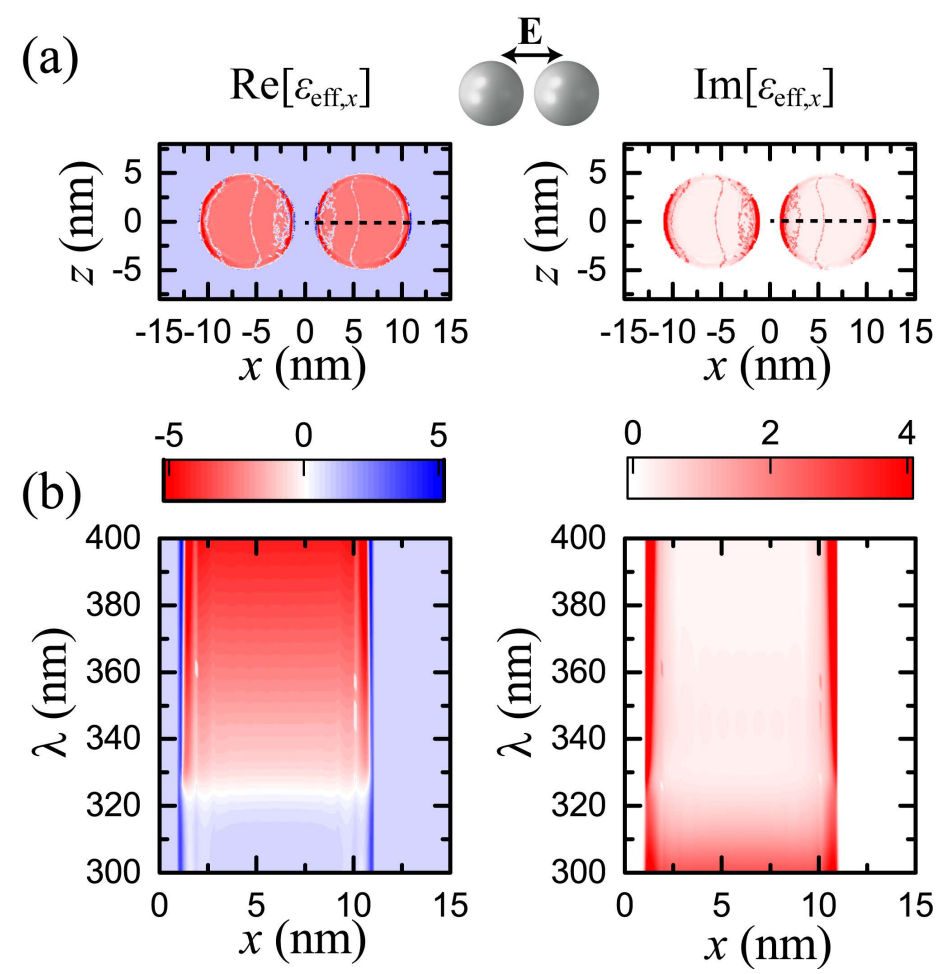

Fig. 5. GNOR calculations for an Ag nanosphere dimer $(R=5 \mathrm{~nm})$ separated by a 2-nm gap, illuminated by a plane wave polarized along the dimer axis ( $x$ axis). (a) Real (left-hand contour) and imaginary (right-hand contour) part of the $x$ component of the effective dielectric function, $\varepsilon_{\text {eff }, x}$, calculated at the resonant wavelength $(\lambda=363 \mathrm{~nm})$ at a plane going through the middle of the particles. (b) Wavelength dependence of the real (left-hand contour) and imaginary (right-hand contour) part of $\varepsilon_{\text {eff,x }}$ along the dashed line in (a). Contour plots in (a) and (b) share common color scales.

rule $\operatorname{as}^{41}$

$$
\varepsilon(r)=\varepsilon_{\mathrm{b}}-\frac{\omega_{\mathrm{p}}^{2}}{\omega^{2}+\mathrm{i} \omega\left[\gamma_{\mathrm{s}}(r)+\gamma_{\mathrm{b}}\right]},
$$

where $\varepsilon_{\mathrm{b}}$ is the background dielectric constant. The surface collision damping and spectral broadening $\gamma_{\mathrm{s}}$ can be viewed as originating from direct transitions between two free-electron states near the Fermi level in the metal, enabled by the collision with a metal wall, which would otherwise be prohibited by the momentum conservation requirement. In that respect, a collision with the metal surface is considered to be no different from any other collision, with say, a phonon or a defect. The transition rate has been estimated using extended in momentum $(k)$-space wavefunctions and a wavevector-dependent Lindhard dielectric function, as described in Refs. 40, 68. Applying the inverse Fourier transform to these results, one can obtain the spatial distribution of the effective dielectric constant, as follows from 

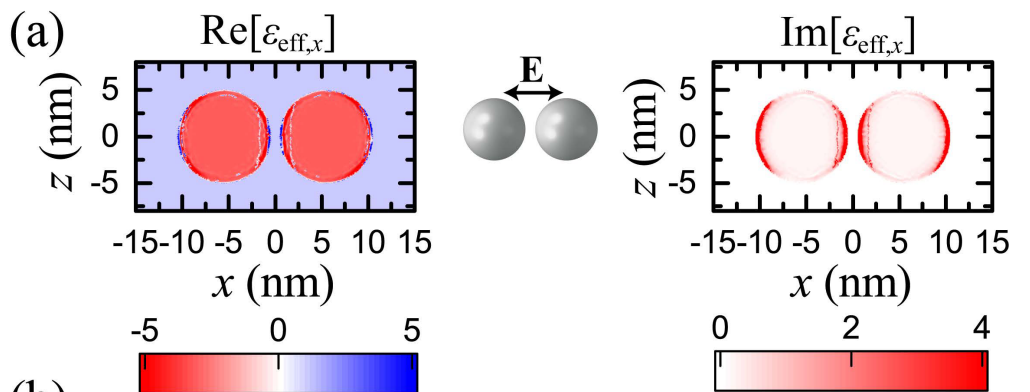

(b)
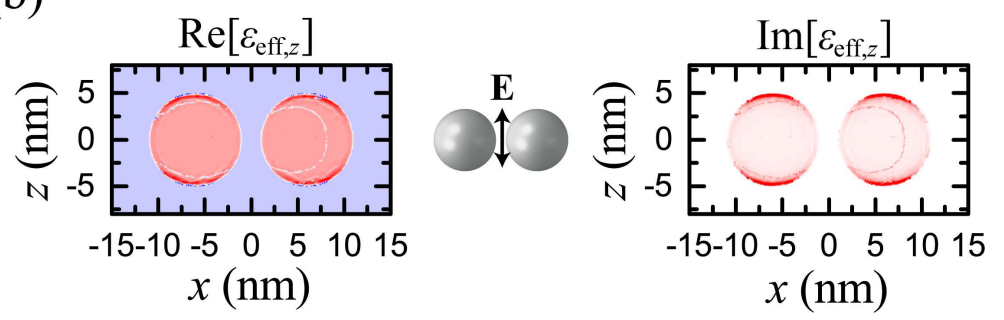

Fig. 6. (a) Real (left-hand contour) and imaginary (right-hand contour) part of the $x$ component of the effective dielectric function, $\varepsilon_{\text {eff, } x}$, calculated at the resonant wavelength $(\lambda=373 \mathrm{~nm})$ for an $\mathrm{Ag}$ nanosphere dimer $(R=5 \mathrm{~nm})$ separated by a 1-nm gap and illuminated by a plane wave polarized along the $x$ axis. (b) Real (left-hand contour) and imaginary (right-hand contour) part of the $z$ component of the effective dielectric function, $\varepsilon_{\mathrm{eff}}, z$, calculated at the resonant wavelength $(\lambda=349 \mathrm{~nm})$ for an $\mathrm{Ag}$ nanosphere dimer $(R=5 \mathrm{~nm})$ separated by a $2-\mathrm{nm}$ gap and illuminated by a plane wave polarized along the $z$ axis. Contour plots in (a) and (b) share common color scales. All calculations are performed with the GNOR theory.

the consideration outlined below. When the plasmon polariton gets absorbed, the electrons (holes) get excited in the wide energy range from the Fermi level $E_{\mathrm{F}}$ to $E_{\mathrm{F}}+\hbar \omega$, i.e. in the wave vector range from $k_{\mathrm{F}}$ (the Fermi wavenumber) to about $k_{\mathrm{F}}+\omega / v_{\mathrm{F}}$. Due to the boundary condition at the surface, all the wavefunctions are in phase and form a wave packet with a full-width at half-maximum of roughly $\Delta L \simeq \pi v_{\mathrm{F}} / \omega=\lambda v_{\mathrm{F}} /(2 c)$, or about $0.7 \mathrm{~nm}$ for $\lambda=370 \mathrm{~nm}$. The shape of positiondependent absorption $\gamma_{\mathrm{s}}(r)$ obviously follows the shape of the excited wave packet, which leads to the effective dielectric function of Eq. (6) exhibiting a spatial dependence, as shown in Fig. 4 for an $R=5 \mathrm{~nm}$ Drude nanosphere $\left(\varepsilon_{\mathrm{b}}=4.1, \hbar \omega_{\mathrm{p}}=9.3\right.$ $\mathrm{eV}, \hbar \gamma_{\mathrm{b}}=0.013 \mathrm{eV}$, and $\hbar \gamma_{\mathrm{s}}$ relaxing at $\left.0.83 \mathrm{eV}^{41}\right)$. One indeed observes a very strong dependence for the imaginary part, and much less pronounced for the real part.

To further explore nonlocal damping in plasmonic nanostructures, we now turn to noble metals, and study in Fig. 5 the case of an Ag nanosphere dimer in air, using the implementation of the GNOR model ${ }^{69}$ to a commercial finite-element solver (Comsol Multiphysics 5.0). ${ }^{70}$ Silver is described here by a Drude model, with the core-electron part of the dielectric function obtained from the experimental dielectric function of Johnson and Christy, ${ }^{71}$ as described in Ref. 46. The two spheres, of radius 
$R=5 \mathrm{~nm}$, are separated by a $2-\mathrm{nm}$ gap, and illuminated by a plane wave polarized along the dimer axis (taken to be the $x$ axis), as shown in the schematics. This dimer is characterized by the excitation of a bonding dimer plasmon, with its resonance at $363 \mathrm{~nm}$. For the specific nanoparticle sizes and separations, nonlocal effects are expected to be important both because of the reduced size of the individual spheres and because of their narrow separation. In Fig. 5(a) we show the real (left-hand contour) and imaginary (right-hand contour) part of the $x$ component of the effective dielectric function, retrieved within GNOR on resonance. Clearly, $\varepsilon_{\text {eff }, x}$ remains almost constant within the bulk of the particles, but large deviations occur near the interfaces, and the imaginary part obtains its largest values in a narrow region of a few Angstrom near the sphere boundaries, supporting again the picture of enhanced Landau damping as the main mechanism behind loss in small plasmonic systems. We note that additional small features inside the particles appear due to numerical instabilities owing to the increased meshing requirements in the finiteelement method. In Fig. 5(b) we plot $\varepsilon_{\text {eff, } x}$ spectra for the same dimer, calculated along a line crossing the right-hand sphere at its middle, along the dashed lines of Fig. 5(a). The calculated dielectric function follows well the experimental one $\mathrm{e}^{71}$ inside the bulk of the particles, with the real part changing sign at about $330 \mathrm{~nm}$, and large nonlocal deviations only appear near the surfaces. It is also worth noticing the behavior of the imaginary part, which is large near the surface for all wavelengths in agreement with our previous discussion, but becomes important also inside the particles for wavelengths shorter than $320 \mathrm{~nm}$, were interband transitions dominate in silver. ${ }^{4}$ Finally, we note that decreasing the gap increases the interaction between the spheres, ${ }^{15}$ and this is accompanied by accordingly higher values of the imaginary part of the effective dielectric function near the surfaces, as shown in Fig. 6(a) for the dimer of Fig. 5 and a 1-nm gap. On the other hand, changing the polarization (taking for example $\mathbf{E}$ along the $z$ axis) leads to a charge accumulation in this direction at the two corresponding sides of the spheres, and Landau damping is mostly experienced there, as shown in Fig. 6(b) for a 2-nm-gap dimer.

\section{Conclusion}

In summary, we have discussed the origin of plasmon damping in flat metal-dielectric interfaces, small metallic nanoparticles, and nanoparticle dimers, in view of the recent GNOR theory for nonlocal plasmonics. Through calculations of the effective dielectric function, using the GNOR model, a generalized Lindhard approach, or through ab initio TDDFT calculations, we have shown that in monomers, and in dimers with separations larger than a few Angstrom and/or a large metal work function, plasmon damping and the corresponding modal broadening are adequately described by nonlocal convection-diffusion theory, which fully captures Landau damping near the metal surfaces. 


\section{Acknowledgements}

C. T. was supported by funding from the People Programme (Marie Curie Actions) of the European Union's Seventh Framework Programme (FP7/2007-2013) under REA grant agreement number 609405 (COFUNDPostdocDTU). N.A.M. and M.W. gratefully acknowledge support from the Villum Foundation via the VKR Centre of Excellence NATEC-II and from the Danish Council for Independent Research (FNU 1323-00087). Center for Nanostructured Graphene (CNG) is financed by the Danish National Research Council (DNRF103). N.A.M. is a Villum Investigator supported by the Villum Foundation.

\section{References}

1. S. A. Maier, Plasmonics: Fundamentals and Applications (Springer, New York, 2007).

2. M. L. Brongersma, Faraday Discuss. 178, 9 (2015).

3. A. Baev, P. N. Prasad, H. Ågren, M. Samoć, and M. Wegener, Phys. Rep. 594, 1 (2015).

4. N. W. Ashcroft and N. D. Mermin, Solid State Physics (Saunders College Publishing, Fort Worth, 1976).

5. L. D. Landau, E. M. Lifshitz, and L. P. Pitaevskii, Electrodynamics of Continuous Media (Butterworth Heinemann, Oxford, 1984).

6. S. I. Bozhevolnyi and N. A. Mortensen, Nanophotonics 6 (2017), DOI: 10.1515/nanoph-2016-0179

7. W. Zhu, R. Esteban, A. G. Borisov, J. J. Baumberg, P. Nordlander, H. J. Lezec, J. Aizpurua, and K. B. Crozier, Nature Commun. 7, 11495 (2016).

8. A. I. Fernández-Domínguez, F. J. García-Vidal, and L. Martín-Moreno, Nature Photon. 11, 8 (2017).

9. S. I. Bozhevolnyi, L. Martín-Moreno, and F. J. García-Vidal, Quantum Plasmonics, Springer Series in Solid-State Sciences 185 (Springer International Publishing, New York, 2017).

10. D. K. Gramotnev and S. I. Bozhevolnyi, Nature Photon. 4, 83 (2010).

11. D. K. Gramotnev and S. I. Bozhevolnyi, Nature Photon. 8, 14 (2014).

12. L. M. Liz-Marzán, Langmuir 22, 32 (2006).

13. E. Hao and G. C. Schatz, J. Chem. Phys. 120, 357 (2004).

14. M. I. Stockman, Phys. Rev. Lett. 93, 137404 (2004).

15. I. Romero, J. Aizpurua, G. Bryant, and F. J. García de Abajo, Opt. Express 14, 9988 (2006).

16. H. Duan, A. I. Fernández-Domínguez, M. Bosman, S. A. Maier, and J. K. W. Yang, Nano Lett. 12, 1683 (2012).

17. A. Wiener, A. I. Fernández-Domínguez, A. P. Horsfield, J. B. Pendry, and S. A. Maier, Nano Lett. 12, 3308 (2012).

18. F. J. García de Abajo, J. Phys. Chem. C 112, 17983 (2008).

19. J. M. McMahon, S. K. Gray, G. C. and Schatz, Phys. Rev. Lett. 103, 097403 (2009).

20. C. David and F. J. García de Abajo, J. Phys. Chem. C 115, 19470 (2011).

21. S. Raza, G. Toscano, A.-P. Jauho, M. Wubs, and N. A. Mortensen, Phys. Rev. B 84, $121412(\mathrm{R})(2011)$.

22. J. Kern, S. Grossmann, N. V. Tarakina, T. Häckel, M. Emmerling, M. Kamp, J.-S. Huang, P. Biagioni, J. Prangsma, and B. Hecht, Nano Lett. 12, 5504 (2012).

23. C. Ciracì, R. T. Hill, J. J. Mock, Y. Urzhumov, A. I. Fernández-Domínguez, S. A. Maier, J. B. Pendry, A. Chilkoti, and D. R. Smith, Science 337, 1072 (2012). 
24. K. J. Savage, M. M. Hawkeye, R. Esteban, A. G. Borisov, J. Aizpurua, and J. J. Baumberg, Nature 491, 574 (2012).

25. J. A. Scholl, A. L. Koh, and J. A. Dionne, Nature 483, 421 (2012).

26. S. Raza, N. Stenger, S. Kadkhodazadeh, S. V. Fischer, N. Kostesha, A.-P. Jauho, A. Burrows, M. Wubs, and N. A. Mortensen Nanophotonics 2, 131 (2013).

27. S. Raza, N. Stenger, A. Pors, T. Holmgaard, S. Kadkhodazadeh, J. B. Wagner, K. Pedersen, M. Wubs, S. I. Bozhevolnyi, and N. A. Mortensen, Nature Commun. 5, 4125 (2014).

28. S. F. Tan, L. Wu, J. K. W. Yang, P. Bai, M. Bosman, and C. A. Nijhuis, Science, 343, 1496 (2014).

29. D. Paria, K. Roy, H. J. Singh, S. Kumar, S. Raghavan, A. Ghosh, and A. Ghosh, Adv. Matter. 27, 1751 (2015).

30. S. Raza, S. Kadkhodazadeh, T. Christensen, M. Di Vece, M. Wubs, N. A. Mortensen, and N. Stenger, Nature Commun. 6, 8788 (2015).

31. H. Shen, L. Chen, L. Ferrari, M.-H. Lin, N. A. Mortensen, S. Gwo, and Z. Liu, Nano Lett. 17, 2234 (2017).

32. S. Raza, S. I. Bozhevolnyi, M. Wubs, and N. A. Mortensen, J. Phys.: Condens. Matter 27, 183204 (2015).

33. G. Toscano, S. Raza, A.-P. Jauho, N. A. Mortensen, and M. Wubs, Opt. Express 20, $4176(2012)$.

34. F. Bloch, Z. Phys. A 81, 363 (1933).

35. R. Ruppin, Phys. Rev. Lett. 31, 1434 (1973).

36. A. D. Boardman, Electromagnetic Surface Modes. Hydrodynamic Theory of PlasmonPolaritons on Plane Surfaces (John Wiley and Sons, Chichester, 1982).

37. J. M. Pitarke, V. M. Silkin, E. V. Chulkov, and P. M. Echenique, Rep. Prog. Phys. 70, 1 (2007).

38. N. A. Mortensen, S. Raza, M. Wubs, T. Søndergaard, and S. I. Bozhevolnyi, Nature Commun. 5, 3809 (2014).

39. A. V. Uskov, I. E. Protsenko, N. A. Mortensen, and E. P. O'Reilly, Plasmonics 9, 185 (2014).

40. J. B. Khurgin, Faraday Discuss. 178, 109 (2015).

41. J. B. Khurgin and G. Sun, in Springer Series in Solid-State Sciences 185: Quantum Plasmonics, ed. S. I. Bozhevolniy et al. (Springer Nature, Cham, 2017), pp. 303-322.

42. W. Yan, M. Wubs, and N. A. Mortensen, Phys. Rev. Lett. 115, 137403 (2015).

43. G. Toscano, S. Raza, W. Yan, C. Jeppesen, S. Xiao, M. Wubs, A.-P. Jauho, S. I. Bozhevolnyi, and N. A. Mortensen, Nanophotonics 2, 161 (2013).

44. U. Kreibig and M. Vollmer, Optical Properties of Metal Clusters (Springer-Verlag, Berlin, 1995).

45. R. Esteban, A. G. Borisov, P. Nordlander, and J. Aizpurua, Nature Commun. 3, 825 (2012).

46. C. Tserkezis, J. R. Maack, Z. Liu, M. Wubs, and N. A. Mortensen, Sci. Rep. 6, 28441 (2016).

47. J. A. Scholl, A. García-Etxarri, A. L. Koh, and J. A. Dionne, Nano Lett. 13, 564 (2013).

48. R. Esteban, A. Zugarramurdi, P. Zhang, P. Nordlander, F. J. García-Vidal, A. G. Borisov, and J. Aizpurua, Faraday Discuss. 178, 151 (2015).

49. U. Hohenester, Phys. Rev. B 91, 205436 (2015).

50. S. Weiss, D. Botkin, D. F. Ogletree, M. Salmeron, and D. S. Chemla, Phys. Stat. Sol. (b) 188, 343 (1995).

51. F. Benz, B. de Nijs, C. Tserkezis, R. Chikkaraddy, D. O. Sigle, L. Pukenas, S. D. 
Evans, J. Aizpurua, and J. J. Baumberg, Opt. Express 23, 33255 (2015).

52. M. Grifoni and P. Hänggi, Phys. Rep. 304, 229 (1998).

53. M. Büttiker, H. Thomas, and A. Prêtre, Phys. Lett. A 180, 364 (1995).

54. U. D. Keil, T. Ha, J. R. Jensen and J. M. Hvam, Appl. Phys. Lett. 72, 3074 (1998).

55. U. Hohenester and C. Draxl, Phys. Rev. B 94, 165418 (2016).

56. A. Vagov, I. A. Larkin, M. D. Croitoru, V. M. Axt, Phys. Rev. B 93, 195414 (2016).

57. T. V. Shahbazyan, Phys. Rev. B. 93, 235431 (2016).

58. X. Li, D. Xiao, and Z. Zhang, New J. Phys. 15, 023011 (2013).

59. D. Jin, Q. Hu, D. Neuhauser, F. von Cube, Y. Yang, R. Sachan, T. S. Luk, D. C. Bell, N. X. Fang, Phys. Rev. Lett. 115, 193901 (2015).

60. P. J. Feibelman, Prog. Surf. Sci. 12, 287 (1982).

61. T. Christensen, W. Yan, A.-P. Jauho, M. Soljačić, and N. A. Mortensen, Phys. Rev. Lett. 118, 157402 (2017).

62. T. V. Teperik, P. Nordlander, J. Aizpurua, A. G. Borisov, Phys. Rev. Lett. 110, 263901 (2013).

63. T. V. Teperik, P. Nordlander, J. Aizpurua, and A. G. Borisov, Opt. Express 21, 27306 (2013).

64. G. Toscano, J. Straubel, A. Kwiatkowski, C. Rockstuhl, F. Evers, H. Xu, N. A. Mortensen, and M. Wubs, Nature Commun. 6, 7132 (2015).

65. W. Yan, Phys. Rev. B 91, 115416 (2015).

66. C. Ciracì and F. Della Sala, Phys. Rev. B 93, 205405 (2016).

67. Z. F. Öztürk, S. Xiao, M. Yan, M. Wubs, A.-P. Jauho, and N. A. Mortensen, J. Nanophoton. 5, 051602 (2011).

68. J. B. Khurgin and G. Sun, Opt. Express 23, 30730 (2015).

69. www.nanopl.org

70. www.comsol.com

71. P. B. Johnson and R. W. Christy, Phys. Rev. B 6, 4370 (1972). 